\title{
A Phase II Study of Amrubicin as a Third-Line or Fourth-Line Chemotherapy for Patients With Non-Small Cell Lung Cancer: Hokkaido Lung Cancer Clinical Study Group Trial (HOT) 0901
}

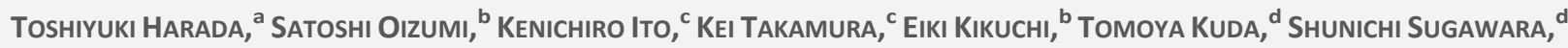

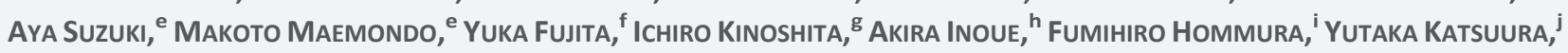 \\ Hirotoshi Dosaka-Akita, ${ }^{\mathrm{g}}$ Hiroshi Isobe, ${ }^{\mathrm{k}}$ Masaharu Nishimura, ${ }^{\mathrm{b}}$ HoKKaido lung CANCer Clinical Study Group

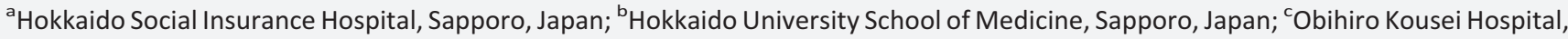

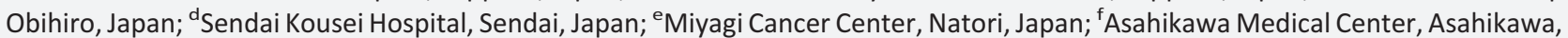 \\ Japan; ${ }^{\mathrm{B}}$ Hokkaido University Graduate School of Medicine, Sapporo, Japan; ${ }^{\mathrm{h}}$ Tohoku University Hospital, Sendai, Japan; 'Sapporo City \\ General Hospital, Sapporo, Japan; 'Saiseikai Fukushima General Hospital, Fukushima, Japan; ${ }^{K}$ Kokka-komuin Kyosai-kumiai Rengokai \\ Sapporo Medical Center, Sapporo, Japan \\ Disclosures of potential conflicts of interest may be found at the end of this article.
}

Key Words. Amrubicin • Chemotherapy • Fourth line • Non-small cell lung cancer • Third line

\section{AbSTRact}

Amrubicin, a third-generation synthetic anthracycline agent, has favorable clinical activity and acceptable toxicity for the treatment of patients with non-small cell lung cancer (NSCLC) and small cell lung cancer. We conducted this study to evaluate the efficacy and safety of amrubicin for advanced NSCLC patients as a third-or fourth-line therapy. Eligible patients had recurrent or refractory advanced NSCLC after second- or third-line therapy. Patients received amrubicin, $35 \mathrm{mg} / \mathrm{m}^{2}$ i.v. on days 1-3 every 3 weeks. The primary endpoint was the disease control rate (DCR). Secondary endpoints were the overall survival (OS) time, progression-free survival (PFS) time, response rate, and toxicity profile. Of the 41 patients enrolled, 26 received amrubicin as a third-line and 15 received it as a fourth-line therapy. The median number of treatment cycles was two (range, 1-9). Objective responses were complete response $(n=0)$, partial response $(n=4)$, stable disease $(n=$
$21)$, progressive disease ( $n=15)$, and not evaluable $(n=1)$, resulting in a DCR of $61.0 \%$ (95\% confidence interval, $46.0 \%-75.9 \%)$. The overall response rate was $9.8 \%(95 \%$ confidence interval, $0.6 \%-18.8 \%)$. The median PFS interval was 3.0 months, median OS time was 12.6 months, and 1-year survival rate was $53.7 \%$. Grade 3 or 4 hematological toxicities were neutropenia (68\%), anemia (12\%), thrombocytopenia (12\%), and febrile neutropenia (17\%). Nonhematological toxicities were mild and reversible. No treatment-related deaths were observed. Amrubicin showed significant clinical activity with manageable toxicities as a third- or fourth-line therapy for patients with advanced NSCLC. This study provides relevant data for routine practice and future prospective trials evaluating third- or fourth-line treatment strategies for patients with advanced NSCLC. The Oncologist 2013;18:439-445

Implications for Practice: There is a paucity of prospective studies that specifically address the role of cytotoxic agents as a thirdline therapy for non-small cell lung cancer (NSCLC) patients. Amrubicin showed significant clinical activity with manageable toxicities as a third- or fourth-line therapy for advanced NSCLC. Amrubicin could be a better candidate in these settings for routine practice.

\section{INTRODUCTION}

Lung cancer is the leading cause of cancer-related death worldwide [1]. First-line therapies, epidermal growth factor receptor (EGFR) tyrosine kinase inhibitors (TKIs) in patients with EGFR mutations, as well as platinum-based chemotherapy in conjunction with third-generation antitumor agents significantly improve survival outcomes and quality of life in patients with advanced non-small cell lung cancer (NSCLC) [2-5]. Despite these favorable outcomes, most pa- tients receiving first-line therapy experience disease progression and require salvage therapy. Second-line therapy also has beneficial effects on survival and quality of life outcomes $[2,6,7]$.

Docetaxel, pemetrexed, gefitinib, and erlotinib are considered standard second-line therapies based on several randomized controlled trials [6-9]. Because of the improved efficacy of first-line, second-line, and maintenance therapy

Correspondence: Toshiyuki Harada, Ph.D., Center for Respiratory Diseases, Hokkaido Social Insurance Hospital, 3-18, 1-8, Nakanoshima, Toyohira-ku, Sapporo 062-8618, Japan. Telephone: 81-11-831-5151; Fax: 81-11-821-3851; E-mail: t-harada@hok-shaho-hsp.jp Received July 19, 2012; accepted for publication November 8, 2012; first published online in The Oncologist Express on February 26, 2013. @AlphaMed Press 1083-7159/ 2013/\$20.00/0 http://dx.doi.org/10.1634/theoncologist.2012-0308 
for NSCLC, a high proportion of patients (26\%-38\%) receive third-line therapy $[10,11]$. Thus, there is an urgent need for new third-line therapy options. To date, there is a paucity of studies that address the role of third-line therapy, and they are primarily retrospective analyses [12-14].

Amrubicin, a completely synthetic 9-amino-anthracycline, is a potent inhibitor of DNA topoisomerase II [15]. A phase II study of amrubicin in both NSCLC and SCLC patients demonstrated promising results and tolerable toxicity $[16,17]$. The clinical significance of amrubicin has recently focused on the treatment of recurring lung cancer. A phase I and a pharmacokinetic study of amrubicin in previously treated NSCLC and SCLC patients recommend an amrubicin dose of $35 \mathrm{mg} / \mathrm{m}^{2}$ per day on three consecutive days every 3 weeks [18]. Amrubicin is a promising third-line therapy agent because it has a different mechanism of action from those of other available anticancer agents.

Currently, there is no prospective study that specifically addresses the role of third-line therapy for NSCLC patients. We therefore conducted a multicenter prospective phase II trial of amrubicin $\left(35 \mathrm{mg} / \mathrm{m}^{2}\right)$ to confirm the efficacy and safety of the drug in NSCLC patients as a third- or fourth-line therapy.

\section{Patients And Methods}

\section{Patient Eligibility}

Eligible patients met the following criteria: histologic or cytologic confirmation of NSCLC, recurrent or refractory disease after two or three previous treatment regimens, measurable disease, an Eastern Cooperative Oncology Group (ECOG) performance status (PS) score of $0-2$, age $\leq 75$ years, adequate bone marrow function (leukocyte count $\geq 3,000 / \mathrm{mm}^{3}$, neutrophil count $\geq 1,500 / \mathrm{mm}^{3}$, platelet count $\geq 100,000 / \mathrm{mm}^{3}$, and hemoglobin content $\geq 9.0 \mathrm{~g} / \mathrm{dL}$ ), adequate function of other organs (total bilirubin concentration $\leq 1.5 \mathrm{mg} / \mathrm{dL}$, aspartate transaminase and alanine transaminase levels $\leq 2.0 \times$ the upper limit of normal, and creatinine clearance $\geq 50 \mathrm{~mL} /$ minute), $\mathrm{P}_{\mathrm{a}} \mathrm{O}_{2} \geq 60$ Torr or $\mathrm{S}_{\mathrm{p}} \mathrm{O}_{2} \geq 95 \%$, left ventricular ejection fraction $\geq 60 \%$ on echocardiography, and a life expectancy $\geq 3$ months.

Patients with previous amrubicin therapy, exceeding the critical dosage in prior anthracycline drug therapy, using corticosteroid or immunosuppressive drugs, with an active infectious disease with serious medical complications (active peptic ulcer, heart disease, diabetes mellitus, or cerebrovascular disease), with radiographic signs of interstitial pneumonia or pulmonary fibrosis, with third-space fluid collection requiring drainage, who were lactating or pregnant, with symptomatic brain metastasis, or with active concomitant malignancy were deemed ineligible.

This study was performed in accordance with the principles of the Declaration of Helsinki and Good Clinical Practice guidelines [19]. The protocol was approved by the institutional review boards of all participating institutions, and all patients provided written informed consent before treatment.

\section{Treatment Plan}

Amrubicin was dissolved in $20 \mathrm{~mL}$ physiological saline and was administered i.v. for $>5$ minutes at a dose of $35 \mathrm{mg} / \mathrm{m}^{2} \cdot$ per day on days 1-3 every 3 weeks. All patients received at least two cycles of treatment unless their disease progressed, unacceptable toxicity occurred, the patient refused further treatment, or the physician decided to discontinue treatment.
Subsequent cycles of treatment were withheld until the following criteria were satisfied: the leukocyte count was $\geq 3,000 / \mathrm{mm}^{3}$, the neutrophil count was $\geq 1,500 / \mathrm{mm}^{3}$, the platelet count was $\geq 100,000 / \mathrm{mm}^{3}$, total bilirubin was $\leq 2.0$ $\mathrm{mg} / \mathrm{dL}$, there was no infection, the ECOG PS score was $\leq 2$, and the grade of any nonhematologic toxicity was $\leq 2$. If these criteria were not satisfied within 36 days after the onset of the last treatment, the patient was removed from the study. The dose of amrubicin was reduced to $30 \mathrm{mg} / \mathrm{m}^{2}$ per day for cases of leukopenia or neutropenia of grade 4 persisting for $>4$ days, thrombocytopenia of grade 4 or requiring platelet transfusion, febrile neutropenia, or nonhematologic toxicity of grade $\geq 3$ (except for anorexia, nausea, or alopecia) during the previous course. If these toxicities occurred after reduction of the amrubicin dose to $30 \mathrm{mg} / \mathrm{m}^{2}$ per day, the dose was further reduced to $25 \mathrm{mg} / \mathrm{m}^{2}$ per day. A third reduction was not permitted, and the protocol treatment was terminated. Use of prophylactic antibiotics was not permitted.

\section{Evaluation}

Baseline assessment included a physical examination; CBC with differential, hepatic, and renal function tests; urinalysis; 12-lead electrocardiogram; echocardiogram; and chest radiography. Visible and palpable tumors were measured during the baseline assessment using chest radiography, computed tomography (CT) scans, or magnetic resonance imaging (MRI) scans (when clinically indicated). During the study, medical history and physical examination results, vital signs, ECOG PS scores, CBCs, and blood chemistries were monitored weekly. Tumor responses were assessed using chest radiography, CT, or MRI (when clinically indicated) at every cycle until disease progression. Unidirectional measurements were adopted on the basis of the Response Evaluation Criteria in Solid Tumors, version 1.0 [20]. A response of $>4$ weeks duration was considered a complete response (CR) or a partial response (PR) and a response of $>6$ weeks from the initiation of chemotherapy was considered stable disease (SD). Clinical response data were confirmed by central review.

Toxicities were assessed according to the National Cancer Institute-Common Toxicity Criteria, version 3.0.

The progression-free survival (PFS) was defined as the time from the date of enrollment to the date of documented progression or death from any cause and was censored at the date of the last follow-up visit for surviving patients who had not progressed. The overall survival (OS) time was defined as the time from the date of enrollment to the date of death or last follow-up. Data for patients without any events were censored on the last date with a nonevent status.

\section{Statistical Analysis}

The primary endpoint was the disease control rate (DCR), defined as the proportion of patients whose best response was a $\mathrm{CR}$, a PR, or SD among all per-protocol patients. Sample size was determined according to the one-arm binomial design devised by the Southwestern Oncology Group. Assuming that a DCR of $50 \%$ in eligible patients indicates potential usefulness, whereas a DCR of $30 \%$ is the lower limit of interest, with $\alpha=$ 0.05 and $\beta=0.20$, the estimated accrual number was 37 patients. Allowing for a patient ineligibility rate of $10 \%$, we planned on enrolling 40 patients in the study. Secondary endpoints were the OS, PFS, objective response rate (ORR), and 
toxicity profiles. Survival curves were estimated using the Kaplan-Meier method. Statistical analyses were performed using JMP 10 (SAS Institute Inc., Cary, NC).

This study is registered with the University Hospital Medical Information Network (UMIN), number UMIN C000002306.

\section{RESULTS}

\section{Patient Characteristics}

From August 2009 to May 2011, 41 patients were enrolled from 10 participating institutions. Patient characteristics are summarized in Table 1 . The median age was 66 years (range, 43-74 years), $70.7 \%$ of patients were male, and most patients $(97.6 \%)$ had a good ECOG PS score of $0-1$. Histologic analysis revealed that 30 patients $(73.2 \%)$ had adenocarcinoma and eight patients (19.5\%) had squamous cell carcinoma. Seven patients (17.1\%) were positive and 26 patients (63.4\%) were negative for the EGFR mutation. Twenty-six patients (63.4\%) received amrubicin as a third-line therapy and 15 patients (36.6\%) received the drug as a fourth-line therapy.

Seven patients (17.1\%) received thoracic surgery and nine patients $(22.0 \%)$ received thoracic radiotherapy. Table 2 shows the content of prior therapeutic regimens. All patients had received a platinum-containing doublet regimen as a firstor second-line therapy. The regimens used in first-line therapy were as follows: platinum-containing doublets in 38 patients $(92.7 \%)$, a single agent in two patients $(4.9 \%)$, and gefitinib in one patient (2.4\%). The regimens used in second-line therapy were as follows: a single agent in 21 patients (51.2\%), platinum-containing doublets in 13 patients (31.7\%), nonplatinum doublets in four patients (9.8\%), and gefitinib in three patients (7.3\%). The regimens used in third-line therapy were as follows: platinum-containing doublets in six patients (14.6\%), a single agent in six patients (14.6\%), and nonplatinum doublets in three patients (7.3\%). Of the seven patients harboring EGFR mutations, three had not received EGFR TKIs before enrollment into this study because of patient refusal or later confirmation of the EGFR mutation.

\section{Treatment Administered}

The median number of treatment cycles was two (range, 1-9 cycles). In all, 30 (73.2\%) patients completed at least two cycles of treatment and 109 treatment cycles in total were delivered overall. The mean relative dose intensity of amrubicin was $91.1 \%$. A reduction in the amrubicin dose was necessary, according to the study protocol, in eight cycles ( $7.3 \%$ of the total cycles). All patients received the first cycle of amrubicin in an inpatient setting to check the safety of administration, and most patients received further cycles of amrubicin in an outpatient setting. Subsequent treatment delay was observed in 29 of 109 cycles (26.6\%). The primary reasons for dose reduction were grade 4 neutropenia (four of all cycles), febrile neutropenia (three of all cycles), and grade 3 headache (one of all cycles). Treatment was discontinued in 11 patients after the first cycle and in 10 patients after the second cycle; reasons for discontinuation included progressive disease (24 patients), toxicity (six patients), completing the scheduled treatment (four patients), patient refusal (two patients), and physician decision (two patients). Following the protocol treatment, 23 (56\%) patients eventually received subsequent therapy: nine (22\%) received a single agent, nine $(22 \%)$ received EGFR TKIs,
Table 1. Patient characteristics

\begin{tabular}{ll}
\hline Characteristic & $n$ of patients \\
\hline Enrolled patients & 41 \\
Age, yrs & 66 \\
Median & $43-74$ \\
Range & \\
Gender & 29 \\
Male & 12 \\
Female & \\
ECOG performance status score & 16 \\
0 & 24 \\
1 & 1 \\
2 & \\
Histological type & 30 \\
Adenocarcinoma & 8 \\
Squamous cell carcinoma & 2 \\
Large cell carcinoma & 1 \\
Other (not specified) & 26 \\
EGFR mutation status & 7 \\
Positive & 26 \\
Negative & \\
Unknown & \\
2 & \\
3 &
\end{tabular}

Abbreviations: ECOG, Eastern Cooperative Oncology Group; EGFR epidermal growth factor receptor.

three $(7 \%)$ received platinum-containing doublets, and two $(5 \%)$ received nonplatinum doublets.

\section{Response and Survival}

Among the 41 assessable patients, there were four PRs and no case of CR, for an overall response rate of $9.8 \%$ (95\% confidence interval [Cl], 0.6\%-18.8\%) (Table 3). Twenty-one patients (51.2\%) had SD, yielding an overall DCR of $61.0 \%(95 \% \mathrm{Cl}, 46.0 \%-75.9 \%)$. Fifteen patients had progressive disease as their best response and the response of one patient could not be confirmed as a result of receipt of subsequent chemotherapy before response evaluation. The lower end of the $95 \% \mathrm{Cl}$ was thus higher than the threshold DCR of $30 \%$, and the primary endpoint was met. We found no significant difference in the ORR or DCR among gender, age, tumor histology, EGFR mutation status, or treatment line, except for the DCR and EGFR mutation status - the DCR was $100 \%$ in seven patients with an EGFR mutation, $46.2 \%$ in 26 patients with wild-type EGFR, and $85.7 \%$ in seven patients with an unknown EGFR status ( $p=.012$ ).

Of the 41 patients, 13 were alive as of May 2012 (>1 year after the last patient was enrolled). With a median follow-up time of 12.6 months, the median PFS and median survival time (MST) for all enrolled patients were 3.0 months ( $95 \% \mathrm{Cl}, 2.0-$ 4.1 months) and 12.6 months ( $95 \% \mathrm{Cl}, 6.8-19.3$ months), respectively (Figs. 1 and 2). The 1-year survival rate was $53.7 \%$ (95\% Cl, 38.4\%-68.9\%). 
Table 2. Characteristics of prior first-line, second-line, and third-line therapies

\begin{tabular}{llll}
\hline Characteristic & First-line therapy & Second-line therapy & Third-line therapy \\
\hline Total & 41 & 41 & 15 \\
Treatment regimen & 38 & 17 & 9 \\
$\quad$ Doublet & 38 & 13 & 6 \\
$\quad$ Platinum-based doublets & 15 & 5 & 2 \\
$\quad$ Paclitaxel based & 9 & 1 & 1 \\
$\quad$ Gemcitabine based & 5 & 0 & 0 \\
Vinorelbine based & 4 & 2 & 0 \\
Docetaxel based & 3 & 4 & 2 \\
Pemetrexed based & 2 & 1 & 1 \\
$\quad$ Other & 0 & 4 & 3 \\
Nonplatinum doublets & 2 & 21 & 6 \\
Single agent & 2 & 15 & 2 \\
Docetaxel & 0 & 6 & 2 \\
Pemetrexed & 0 & 0 & 2 \\
Vinorelbine & 1 & 3 & 0 \\
Gefitinib & & & \\
\hline
\end{tabular}

Table 3. Overall response rate for patients treated with amrubicin as determined by central review

\begin{tabular}{lccc}
\hline Response & n of patients & $\%$ & 95\% Cl \\
\hline Complete response & 0 & 0 & 9.8 \\
Partial response & 4 & 51.2 & 36.6 \\
Stable disease & 21 & 2.4 & $0.6 \%-18.8 \%$ \\
Progressive disease & 15 & 9.8 & $46.0 \%-75.9 \%$ \\
Not evaluable & 1 & 61.0 & \\
Overall response rate & 4 & & \\
Disease control rate & 25 & & \\
\hline
\end{tabular}

Abbreviation: $\mathrm{Cl}$, confidence interval.

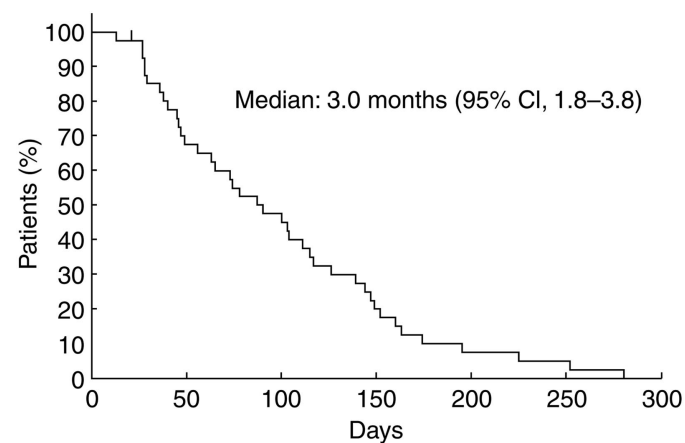

Figure 1. Kaplan-Meier analysis of progression-free survival for all 41 treated patients.

Abbreviation: $\mathrm{Cl}$, confidence interval.

\section{Toxicity}

All 41 treated patients were assessed for toxicity. Table 4 summarizes the hematological and nonhematological toxicities. With regard to hematological toxicities, $68 \%$ of patients experienced grade 3 or 4 neutropenia and $17 \%$ developed febrile neutropenia. Nineteen patients (46\%) were treated with GCSF for 1-11 days during the first treatment cycle because of neutropenia. Although no serious hematologic events were observed, grade 3 or 4 thrombocytopenia was observed in five patients (12\%; one received a platelet transfusion) and ane-

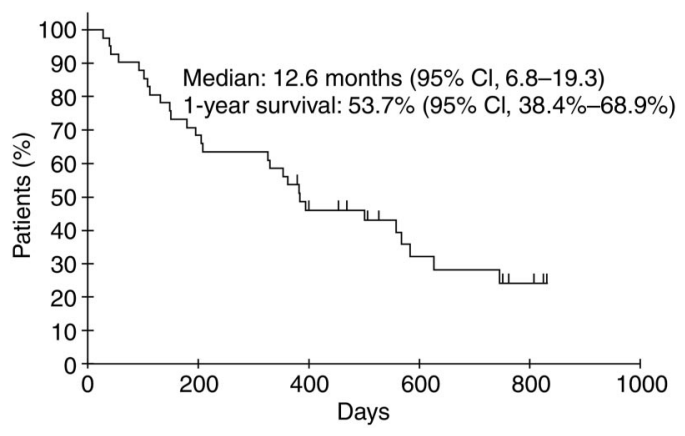

Figure 2. Kaplan-Meier analysis of overall survival for all 41 treated patients.

Abbreviation: $\mathrm{Cl}$, confidence interval.

mia was observed in five patients (12\%; two received a packed RBC transfusion). The most common nonhematologic toxicities of grade 3 or 4 were anorexia (12\%), infection (10\%), nausea or vomiting $(10 \%)$, diarrhea $(2 \%)$, stomatitis $(2 \%)$, and pneumonitis (2\%). Most nonhematologic toxicities were mild and reversible. Neither cardiac toxicity nor treatment-related deaths were observed in this study.

\section{DisCUSSION}

This is the first prospective phase II study designed to evaluate the efficacy and safety of a cytotoxic agent as a third- or 
Table 4. Toxicity for all cycles

\begin{tabular}{|c|c|c|c|c|c|c|}
\hline \multirow[b]{2}{*}{ Toxicity } & \multicolumn{4}{|c|}{ Grade } & \multicolumn{2}{|c|}{ Grade $\geq 3$} \\
\hline & 1 & 2 & 3 & 4 & $n$ & $\%$ \\
\hline \multicolumn{7}{|l|}{ Hematologic } \\
\hline Leukopenia & 7 & 5 & 17 & 5 & 22 & 53.7 \\
\hline Neutropenia & 4 & 5 & 8 & 20 & 28 & 68.3 \\
\hline Anemia & 23 & 6 & 4 & 1 & 5 & 12.2 \\
\hline Thrombocytopenia & 6 & 3 & 4 & 1 & 5 & 12.2 \\
\hline Febrile neutropenia & 0 & 0 & 7 & 0 & 7 & 17.1 \\
\hline \multicolumn{7}{|l|}{ Nonhematologic } \\
\hline Nausea/vomiting & 15 & 4 & 4 & 0 & 4 & 9.8 \\
\hline Anorexia & 10 & 5 & 5 & 0 & 5 & 12.2 \\
\hline Diarrhea & 2 & 0 & 1 & 0 & 1 & 2.4 \\
\hline Stomatitis & 0 & 0 & 1 & 0 & 1 & 2.4 \\
\hline Pneumonitis & 1 & 2 & 1 & 0 & 1 & 2.4 \\
\hline Infection & 0 & 1 & 4 & 0 & 4 & 9.8 \\
\hline Fever & 0 & 1 & 0 & 0 & 0 & 0 \\
\hline Fatigue & 1 & 1 & 0 & 0 & 0 & 0 \\
\hline Liver dysfunction & 7 & 0 & 0 & 0 & 0 & 0 \\
\hline
\end{tabular}

fourth-line chemotherapy for patients with advanced NSCLC. Our study demonstrated the efficacy of amrubicin as shown by the ORR of $9.8 \%$, DCR of $61.0 \%$, median PFS of 3.0 months, median OS of 12.6 months, and 1-year survival rate of $53.7 \%$ in 41 patients. Although common, hematological toxicities were manageable and nonhematological toxicities were mild and reversible. Previous phase III trials for second- or third-line therapy of NSCLC patients have reported ORRs of 7.6\%-9.1\%, median OS of 6.7-8.3 months, and 1-year survival rates of $29.7 \%-34 \%[6-9,21]$. Amrubicin is a potent inhibitor of topoisomerase II, with a different mechanism of action from those of the currently available active cytotoxic agents for advanced NSCLC [15].

Several studies have evaluated the efficacy and safety of amrubicin for patients with advanced NSCLC. With regard to the dose of amrubicin, favorable results with tolerable toxicity were demonstrated with $45 \mathrm{mg} / \mathrm{m}^{2}$ per day on three consecutive days every 3 weeks for first-line therapy [17, 22], 40 $\mathrm{mg} / \mathrm{m}^{2}$ for second-line therapy (West Japan Thoracic Oncology Group [WJTOG]0401 trial) [23], $35 \mathrm{mg} / \mathrm{m}^{2}$ for primarily second-line therapy and for a small number of patients treated with third-line therapy [24], and $35 \mathrm{mg} / \mathrm{m}^{2}$ or 40 $\mathrm{mg} / \mathrm{m}^{2}$ for third or subsequent lines of therapy [25]. Based upon these results, we conducted a prospective phase II trial of amrubicin at a dose of $35 \mathrm{mg} / \mathrm{m}^{2}$ in NSCLC patients as a third-or fourth-line therapy.

The incidence of febrile neutropenia was certainly high in this study ( $17 \%$, seven of 41 patients). Five patients developed febrile neutropenia in the first cycle, one patient developed this toxicity in the second cycle, and one patient developed this toxicity in the fourth cycle. A previous phase Il study of amrubicin at $40 \mathrm{mg} / \mathrm{m}^{2}$ for the second-line therapy of NSCLC patients (the WJTOG0401 trial) reported that $29.5 \%$ of the patients developed febrile neutropenia [23]. Thus, the possible reasons for the higher incidence of febrile neutropenia in our study are as follows: the $35-\mathrm{mg} / \mathrm{m}^{2}$ dose of amrubicin might be formidable in the third-or fourth-line setting and neither the prophylactic use of G-CSF nor the prophylactic use of antibiotics was allowed. In addition, an adverse event of particular concern related to amrubicin administration is cardiac toxicity, the incidence of which was $3.2 \%$ in previous trials [17, 22]. For safety reasons, this study allowed the enrollment of only patients with a left ventricular ejection fraction of $60 \%$ as determined by echocardiography. No cardiac toxicity was observed in our trial, and there were no treatment-related deaths in this study.

The number of patients who need third-line therapy is increasing, and third-line therapy represents a clinical problem for advanced NSCLC treatment. However, there is no standard definition for third-line therapy because the population of patients who could be grouped as potential candidates is heterogeneous [13] and clinical trial information regarding this population is sparse. Retrospective analyses from three institutions reported that $20.3 \%, 28.2 \%$, and $38.4 \%$ of patients received third-line chemotherapy in clinical practice $[11,14,26]$. Those analyses showed that the ORR was 5.6\%-17.0\%, the DCR was $34.4 \%-44.4 \%$, the median PFS was 2.4 months, and the median OS was 5.8-12.0 months. Patients who received third-line therapy benefited over those who did not.

In the second-line or later settings, the MST is not associated with the ORR, but is associated with the DCR [27]. Because amrubicin has a different mechanism of action from those of other anticancer agents and is associated with a high DCR with tolerable toxicity $[15,17]$, we conducted the present study in which the DCR was chosen as the primary endpoint. Based on a DCR of $33 \%-56 \%$ in previous phase III trials of second- and third-line therapy of NSCLC patients [7, 8, 28, 29] and the third-and fourth-line settings of this study, we assumed that a DCR of $50 \%$ was a desirable target and that a DCR of $30 \%$ was irrelevant. The lower end of the $95 \% \mathrm{Cl}$ in the present study was higher than the threshold DCR of $30 \%$, and the primary endpoint was met. Single-agent chemotherapy and 
EGFR TKI therapy had advantages over doublet chemotherapy in prolonging PFS and decreasing toxicity when used in thirdline therapy [26]. In a BR.21 trial, erlotinib demonstrated survival benefits when administered as a second- or third-line therapy, compared with placebo (OS, 6.7 months vs. 4.7 months, respectively) [7]. That landmark trial included $49 \%$ of patients receiving third-line therapy; thus, treatment with erlotinib is recommended as a third-line therapy for patients with a PS of $0-3$ who have not received prior EGFR TKIs. These data are not sufficient to make a recommendation for or against using a cytotoxic agent as a third-line therapy. However, there is no prospective trial of cytotoxic agents in that setting, which encouraged us to conduct the present study.

Similar to any phase II study, this analysis has several limitations. All patients enrolled in this study had an ECOG PS of $0-1$, except for one patient with PS score of 2 . Thus, these patients may not be representative of the population of patients with NSCLC who receive third- or fourth-line therapy in clinical practice. Additionally, seven (17\%) patients harboring an EGFR mutation had an excellent prognosis (median OS, 16.7 months; range, 10.8-27.2 months). The survival outcome might have been influenced by the good health condition of these patients or by the indolent nature of the patients' disease. Therefore, further evaluation is warranted to implement this regimen into daily clinical practice as a third- or fourth-line therapy.

\section{CONCLUSION}

In conclusion, amrubicin is a promising agent associated with effective disease control and manageable toxicity as a third-or fourth-line treatment for patients with advanced NSCLC. This study provides relevant data for routine practice and future prospective trials evaluating third- or fourth-line treatment strategies for patients with advanced NSCLC.

\section{ACKNOWLEDGMENTS}

This study is registered with University Hospital Medical Information Network Clinical Trial Registry (http://www.umin. ac.jp/ctr/index.htm; identification number, UMIN c000002306).

\section{Author Contributions}

Conception/Design: Toshiyuki Harada, Satoshi Oizumi

Provision of study material or patients: Toshiyuki Harada, Satoshi Oizumi, Kenichiro Ito, Kei Takamura, Eiki Kikuchi, Tomoya Kuda, Shunichi Sugawara, Aya Suzuki, Makoto Maemondo, Yuka Fujita, Ichiro Kinoshita, Akira Inoue, Fumihiro Hommura, Yutaka Katsuura, Hirotoshi Dosaka-Akita, Hiroshi Isobe, Masaharu Nishimura

Collection and/or assembly of data: Toshiyuki Harada, Satoshi Oizumi, Kenichiro Ito, Kei Takamura, Eiki Kikuchi, Tomoya Kuda, Shunichi Sugawara, Aya Suzuki, Makoto Maemondo, Yuka Fujita, Ichiro Kinoshita, Akira Inoue, Fumihiro Hommura, Yutaka Katsuura, Hirotoshi Dosaka-Akita, Hiroshi Isobe, Masaharu Nishimura

Manuscript writing: Toshiyuki Harada, Satoshi Oizumi, Fumihiro Hommura, Yutaka Katsuura

Final approval of manuscript: Toshiyuki Harada, Satoshi Oizumi, Kenichiro Ito, Kei Takamura, Eiki Kikuchi, Tomoya Kuda, Shunichi Sugawara, Aya Suzuki, Makoto Maemondo, Yuka Fujita, Ichiro Kinoshita, Akira Inoue, Hirotoshi Dosaka-Akita, Hiroshi Isobe, Masaharu Nishimura

\section{DisClOSURES}

The author indicated no financial relationships.

\section{REFERENCES}

1. Jemal A, Siegel R, Ward E et al. Cancer statistics, 2009. CA Cancer J Clin 2009;59:225-249.

2. Pfister DG, Johnson DH, Azzoli CG et al.; American Society of Clinical Oncology. American Society of Clinical Oncology treatment of unresectable nonsmall-cell lung cancer guideline: Update 2003. J Clin Oncol 2004;22:330-353.

3. Schiller JH, Harrington D, Belani CP et al.; Eastern Cooperative Oncology Group. Comparison of four chemotherapy regimens for advanced nonsmall-cell lung cancer. N Engl J Med 2002;346:9298.

4. NSCLC Meta-Analyses Collaborative Group. Chemotherapy in addition to supportive care improves survival in advanced non-small-cell lung cancer: A systematic review and meta-analysis of individual patient data from 16 randomized controlled trials. J Clin Oncol 2008;26:4617-4625.

5. Oizumi S, Kobayashi K, Inoue A et al. Quality of life with gefitinib in patients with EGFR-mutated non-small cell lung cancer: Quality of life analysis of North East Japan Study Group 002 Trial. The Oncologist 2012;17:863-887.

6. Shepherd FA, Dancey J, Ramlau R et al. Prospective randomized trial of docetaxel versus best supportive care in patients with non-small-cell lung cancer previously treated with platinum-based chemotherapy. J Clin Oncol 2000;18:2095-2103.

7. Shepherd FA, Rodrigues Pereira J, Ciuleanu T et al.; National Cancer Institute of Canada Clinical Trials Group. Erlotinib in previously treated non-smallcell lung cancer. N Engl J Med 2005;353:123-132.

8. Hanna N, Shepherd FA, Fossella FV et al. Ran- domized phase III trial of pemetrexed versus docetaxel in patients with non-small-cell lung cancer previously treated with chemotherapy. J Clin Oncol 2004;22:1589-1597.

9. Kim ES, Hirsh V, Mok T et al. Gefitinib versus docetaxel in previously treated non-small-cell lung cancer (INTEREST): A randomised phase III trial. Lancet 2008;372:1809-1818.

10. Murillo JR Jr, Koeller J. Chemotherapy given near the end of life by community oncologists for advanced non-small cell lung cancer. The Oncologist 2006;11:1095-1099.

11. Asahina $\mathrm{H}$, Sekine I, Horinouchi $\mathrm{H}$ et al. Retrospective analysis of third-line and fourth-line chemotherapy for advanced non-small-cell lung cancer. Clin Lung Cancer 2012;13:39-43.

12. de Marinis F, Grossi F. Clinical evidence for second- and third-line treatment options in advanced non-small cell lung cancer. The Oncologist 2008; 13(suppl 1):14-20.

13. Azzoli CG, Baker S Jr, Temin S et al.; American Society of Clinical Oncology. American Society of Clinical Oncology clinical practice guideline update on chemotherapy for stage IV non-small-cell lung cancer. J Clin Oncol 2009;27:6251-6266.

14. Girard N, Jacoulet P, Gainet M et al. Third-line chemotherapy in advanced non-small cell lung cancer: Identifying the candidates for routine practice. J Thorac Oncol 2009;4:1544-1549.

15. Hanada M, Mizuno S, Fukushima A et al. A new antitumor agent amrubicin induces cell growth inhibition by stabilizing topoisomerase II-DNA complex. Jpn J Cancer Res 1998;89:1229-1238.
16. Inoue A, Sugawara S, Yamazaki K et al. Randomized phase II trial comparing amrubicin with topotecan in patients with previously treated smallcell lung cancer: North Japan Lung Cancer Study Group Trial 0402. J Clin Oncol 2008;26:5401-5406.

17. Sawa T, Yana T, Takada M et al. Multicenter phase II study of amrubicin, 9-amino-anthracycline, in patients with advanced non-small-cell lung cancer (study 1): West Japan Thoracic Oncology Group (WJTOG) trial. Invest New Drugs 2006; 24:151-158.

18. Okamoto I, Hamada A, Matsunaga $Y$ et al. Phase I and pharmacokinetic study of amrubicin, a synthetic 9-aminoanthracycline, in patients with refractory or relapsed lung cancer. Cancer Chemother Pharmacol 2006;57:282-288.

19. World Medical Association Declaration of Helsinki. Recommendations guiding physicians in biomedical research involving human subjects. JAMA 1997;277:925-926.

20. Therasse $P$, Arbuck SG, Eisenhauer EA et al. New guidelines to evaluate the response to treatment in solid tumors. European Organization for Research and Treatment of Cancer, National Cancer Institute of the United States, National Cancer Institute of Canada. J Natl Cancer Inst 2000;92: 205-216.

21. Fossella FV, DeVore R, Kerr RN et al. Randomized phase III trial of docetaxel versus vinorelbine or ifosfamide in patients with advanced non-small-cell lung cancer previously treated with platinum-containing chemotherapy regimens. The TAX 320 NonSmall Cell Lung Cancer Study Group. J Clin Oncol 2000;18:2354-2362. 
22. Takeda K, Takifuji N, Negoro S et al. Phase II study of amrubicin, 9-amino-anthracycline, in patients with advanced non-small-cell lung cancer: A West Japan Thoracic Oncology Group (WJTOG) study. Invest New Drugs 2007;25:377-383.

23. Kaneda H, Okamoto I, Hayashi H et al. West Ja pan Thoracic Oncology Group. Phase II trial of amru bicin for second-line treatment of advanced nonsmall cell lung cancer: Results of the West Japan Thoracic Oncology Group trial (WJTOG0401). J Tho rac Oncol 2010;5:105-109.

24. Kaira K, Sunaga N, Tomizawa Y et al. A phase II study of amrubicin, a synthetic 9-aminoanthracy- cline, in patients with previously treated lung cancer. Lung Cancer 2010;69:99-104

25. Igawa S, Takahashi T, Nakamura Y et al. Efficacy of amrubicin for non-small cell lung cancer af ter failure of two or more prior chemotherapy regimens. Anticancer Res 2008;28:3855-3858.

26. Song Z, Yu Y, Chen Z et al. Third-line therapy for advanced non-small-cell lung cancer patients: Feasible drugs for feasible patients. Med Oncol 2011 28(suppl 1):S605-S612.

27. Hotta K, Fujiwara Y, Kiura K et al. Relationship between response and survival in more than 50,000 patients with advanced non-small cell lung cancer treated with systemic chemotherapy in 143 phase III trials. J Thorac Oncol 2007;2:402407.

28. Maruyama R, Nishiwaki $Y$, Tamura $T$ et al. Phase III study, V-15-32, of gefitinib versus docetaxel in previously treated Japanese patients with non-small-cell lung cancer. J Clin Oncol 2008;26: 4244-4252.

29. Ohe $Y$, Ichinose $Y$, Nakagawa $K$ et al. Efficacy and safety of two doses of pemetrexed supplemented with folic acid and vitamin B12 in previously treated patients with non-small cell lung cancer. Clin Cancer Res 2008;14:4206-4212. 